\title{
Available Bandwidth Detection with Improved Transport Control Algorithm for
}

\section{Heterogeneous Networks}

\author{
Qi Fang \\ College of Information Science \\ and Engineering \\ Central South University, \\ Changsha, P. R. China \\ csqifang@mail.csu.edu.cn
}

\author{
Weijia Jia \\ City University of Hong Kong \\ 83 Tat Chee Ave, Kowloon \\ Hong Kong, SAR China \\ itjia@cityu.edu.hk
}

Jie Wu

Dept. of Comp. Sci \& Eng.

Florida Atlantic University

Florida, USA

jie@cse.fau.edu

\begin{abstract}
In this paper, we propose a transport control performance improvement algorithm in heterogeneous networks. This paper discusses some novel algorithms to estimate the available bandwidth and to distinguish congestion error losses from wireless transmission error losses. The main characteristics of the algorithms is that it is non intrusive, i.e., it does not need to inject the traffic into the network in order to estimate the available bandwidth. Thus, our algorithms proposed can improve the throughput and can also be applied to different types of networks adaptively. We report experimental results to efficiency of the proposed algorithm.
\end{abstract}

\section{Introduction}

Internetworking with wired and wireless links in a heterogeneous environment offers a way to support mobile computing. In the heterogeneous network, the packet loss which dose not result from congestion but occurs frequently due to degradation of radio environment such as fading and handoff[1]. The Transmission control Protocol (TCP) performance degrades greatly in the heterogeneous environment.

* The work is supported by City University of Hong Kong strategic grants 7001709 and 7001587.
The approached to improving TCP performance can be classified into (1) link-layer protocols, split-connection protocols, and (3) end-to-end protocols[2].

Section 2.1 presents a new effective method using packet loss distinguishing. Section 2.2 presents a new available bandwidth detection mechanism in wireless setting. Section 2.3 presents the method of estimating the available bandwidth used as setting up the parameters of congestion control. In section 3 we describe the transport control algorithm using the bandwidth estimation and the packet loss discrimination for improving the transport over heterogeneous networks. Performance evaluation is presented in Section 4 and Section 5 concludes the paper.

\section{Improved transport control procedure}

In order to prevent too much decrease of throughput when fast retransmission or timeout occurs, the algorithm distinguishes congestion error losses from wireless transmission error losses. When there is no congestion and the packet loss is mainly caused by the wireless link, we estimating the wireless available bandwidth. When the wireless channel has not enough unutilized bandwidth, the algorithm rejects new traffic communication.

\subsection{Distinguishing the cause of packet loss}

In this section, we present our scheme and measure its ability to distinguish congestion error losses from 
wireless transmission error losses in details.

By comparing the estimated recent throughput with the instantaneous sending rate obtained from congestion window (cwin), we can estimate whether there is congestion in the mixed networks and measure the path congestion level. The difference between the instantaneous sending rate (as obtained from cwin) and the achieved throughput clearly feeds the bottleneck queue, thus revealing that the path is becoming congested.

Let $t_{k}$ be the time instant at which the $k_{t h}$ ACK is received at the sender. The value of RTT can be obtained the estimation in the protocol.

Let $T H_{\text {sample }}(\mathrm{k})$ be a sample of throughput during the previous RTT when the $k$ th ACK is received at the sender. $T H_{\text {sample }}(\mathrm{k})$ is calculated as:

$$
T H_{\text {sample }}(\mathrm{k})=\frac{\sum_{i \in R T} d_{j}}{R T T}
$$

Where $\sum_{j \in T_{k}} d_{j}$ is the amount of data that have been reported delivered by the $j$ th ACK during the previous RTT. We employ a constant-gain ( $\beta=0.6$ ) filter to calculate the recent throughput as:

$$
T H_{\text {estimated }}(k)=T H_{\text {estimated }}(k-1) \times \beta+T H_{\text {sample }}(k) \times(1-\beta)
$$

From the character of throughput and parameter $c$ win, it can be known that if $T H_{\text {estimated }}(k) \times \mathrm{RTT}$ is larger than the current $c$ win value, a path is not congested.

\subsection{Estimating wireless bandwidth}

In a wireless setting, a fairly accurate knowledge of available bandwidth has the additional advantage that it can be combined with loss rate information to help to distinguish channel and congestion errors.

We define the available bandwidth over one link as the link bandwidth minus the unutilized bandwidth. Typical method of end-to-end probe measurement algorithms for available bandwidth is the packet pair approach [3]. Several enhancements such as Pathload [4] and TOPP [5]have been proposed to estimate the bandwidth available along a path. Here we propose an approach to dynamically estimate the wireless link bandwidth using signal-to-noise ratio (SNR) information.

The signal-to-noise ratio (SNR) $\gamma$ is a variable effected by fading. And a corresponding measure in a wireless communication environment is the received bit-energy-to-noise ratio $\gamma$ is in direct proportion to the square of Rayleigh distributed random process envelop in the flat fading channel model.

$$
p_{\gamma}(\gamma)=\frac{1}{\gamma} e^{-\gamma / \gamma_{0}}, \gamma_{0}=E(\gamma)
$$

For example in DPSK without fading, the bit error rate is expressed in [7] as

$$
\mathrm{BER}=\frac{1}{2} \exp (-\gamma)
$$

Then, in DPSK with Rayleigh fading, the average bit error rate can be denoted as

BER_ave $=\int_{0}^{\infty} \frac{1}{\gamma} e^{-\gamma / \gamma_{0}} \frac{1}{2} \exp (-\gamma) d \gamma=\frac{1}{2+\gamma_{0}}$

The relationship between packet error rate (PER) and bit error rate (BER) depends on the channel coding scheme. Assume that two samples of the process are almost independent (fast fading), or in other words, there is no error-correction coding applied and the number of bits in a packet $n$.

$\operatorname{PER}(\mathrm{t})=1-(1-\mathrm{BER}(\mathrm{t}))^{n}$

Assume that $t p$ and $t a$ are the times to transmit a packet and an ACK respectively. Furthermore, tproc and tprop are the packet processing time at the end hosts and the packet propagation time across the channel, respectively. Let to denote the timeout value of the timer and $T w$ be the time that spending during binary exponential backoff in CSMA/CA MAC protocol. Assume the capacity of the wireless link is $C$. The channel efficiency expressed in [7] can be written as:

effi_ink $=\frac{E\left(t_{p}\right)(1-P E R)}{(1-P E R) \times E\left(t_{p}+t_{a}+2 t_{p r o c}+2 t_{p r p p}\right)+P E R \times E\left(t_{p}+t_{o}+t_{p r o c}+t_{p q p}\right)+E\left(t_{w}\right)}$

Thus, we can estimate the available bandwidth with Eq.(8) as

Avai_bandwidth_ave $=e f f i \_l i n k \times C$ 
The estimation scheme is passive measurement, not using any probing messages that may interfere with the networks. The method need not wait too long for data convergence.

\subsection{Estimate the available bandwidth}

In this section, we aim to use the information of available bandwidth for setting up the parameters $c$ win and threshold (ssthresh). We consider the constant-bit-rate traffic (CBR) source and its idle time and busy time are exponentially distributed with mean $0.1 \mathrm{sec}$. When we set the time interval $T_{k}$ over which the bandwidth sample is calculated, the value should not exceed $0.1 \mathrm{sec}$. Moreover, the value should exceed the $A C K$ inter-arrival time.

Let $B W_{\text {sample }}(k)$ be the bandwidth sample, and $B W_{\text {estimated }}(k)$ be estimate of the bandwidth at time $t_{k}$. If an ACK is received at the source at time $t_{k}, d_{k}$ means the corresponding amount of data that have been received by the TCP receiver. Then $B W_{\text {sample }}(k)$ can be expressed as

$$
B W_{\text {sample }}(k)=\frac{\sum_{j \in T_{k}} d_{j}}{T_{k}}
$$

Where $\sum_{j \in T_{k}} d_{j}$ means the amount of data have been delivered by the $j$ th ACK during the calculating the bandwidth sample at time $T_{k}$.

The bandwidth estimation is computed using a time varying coefficient, exponentially-weighted moving average (EWMA) filter. The estimation of the bandwidth of the network can be expressed as follows:

$$
\begin{gathered}
B W_{\text {estimated }}(k)=B W_{\text {estimated }}(k-1) \times \lambda_{k}+B W_{\text {sample }}(k) \times\left(1-\lambda_{k}\right)(10) \\
\text { Where } \lambda_{k}=\frac{2 \alpha_{k}-\Delta t_{k}}{2 \alpha_{k}+\Delta t_{k}} \text {, and } a_{k} \text { is a filter }
\end{gathered}
$$
parameter which determines the filter gain, and varies over the time adapting a path conditions. According to the Shannon sampling theorem, in order to sample a signal with bandwidth $1 / a_{k}$, a sampling interval less or equal to $c\left(1+\gamma_{k}\right)$. In Section 2.2, it can be known that $c$ is the capacity of the wireless link, and $\gamma_{k}$ is signal-to-noise ratio (SNR) when the $k$ th ACK is received at the source at time $t_{k}$.

Otherwise, because the interval between the consecutive acknowledgements $\Delta t_{k}$ are likely to wary between the smallest the bottleneck capacity, $\alpha_{k}$ should be larger than RTT. From the discussion above, the filter parameter $\alpha_{k}$ is set as

$\alpha_{k}={ }_{\max }\left\{\frac{1}{c\left(1+\gamma_{k}\right)}, R T T+N \times R T T\right\}$

The value of $N$ should be positive integer.

\section{Improved transport control algorithm}

In this section we describe how the improving transport control algorithm uses the bandwidth estimation and the packet loss discrimination in Fig1. After indicating packet loss, the improving transport control algorithm distinguish congestion error losses from wireless transmission error losses, then excused different procedure in different situation, and the algorithm describe how to use the information of available bandwidth for setting up the parameters $c$ win and ssthresh.

\section{Algorithm 1:}

1. If send received $n$ DUPACKS

2. If $T H_{\text {estimated }}(k) \times \mathrm{RTT}$ is much larger than cwin,

/* it means "no congestion in the path and the packet loss is mainly caused by the wireless link"*/

3. Then enhance the sending power of base station; estimate Avai_bandwidth_ave from the SNR ;

4. If Avai_bandwidth_ave $<$ threshold excuse admission control ;

5. Else if $T H_{\text {estimated }}(k)$ is descend distinctly

6. ssthresh $\left.=B W_{\text {estimated }}(k) \times \mathrm{RTTmin}\right) / \mathrm{max} \_$seg_size;

7. If (cwin>ssthresh), cwin=ssrhresh;

Fig 1. Improving transport control algorithm

The calculation procedures of $T H_{\text {estimated }}(k)$, Avai_bandwidth_ave and $B W_{\text {estimated }}(k)$ are described in Sections 2.1, 2.2 and 2.3, respectively. And max_seg_size means max transport segment size. 


\section{Performance Evaluations}

In this section, we derived simulation results by introducing some extensions to ns-2 [7]. The TCP transmitter and the the Base station (BS) are connected through two-step wired links representing the fixed portion of the network. The wireless link is assumed to connect the base station to a destination mobile terminal.

We compare the performance of our proposed algorithm to that of Reno and Vegas. We ran simulation with the wired portion propagation time varying from 0 to $200 \mathrm{~ms}$. We assume that the wireless portion of the network is a very short $2 \mathrm{Mbps}$ wireless link with a propagation of propagation time of $0.01 \mathrm{~ms}$.

As showed in the result in Figure 2, when the propagation time is small, all algorithms are equally effective. But the proposed algorithm improves the performance of transport greatly at time $98 \mathrm{~ms}$. As the propagation time increased continually, the throughput of all the algorithms decreased.

We ran simulation with the wireless portion transmission speed of the bottleneck link varying from 2 mbps to 8 mbps and with the wired portion 10 mbps. Simulation results in Figure 3 show that the throughput of all algorithms increases significantly as the bottleneck link transmission speed increases. But as the increase of transmission speed of the bottleneck link, the proposed algorithm is more effective than Reno and Vegas.

\section{Conclusions}

In this paper, we have described a new bandwidth estimation technique and a method that distinguishing the congestion error losses from wireless transmission error losses method. But with the link layer recovery fair sharing being enforced, the algorithm performs poorly when random packet loss rate exceeds some percent. The above mentioned bound shows that the throughput degradation in noisy channels is unavoidable and that the main factor limiting performance is random packet loss.

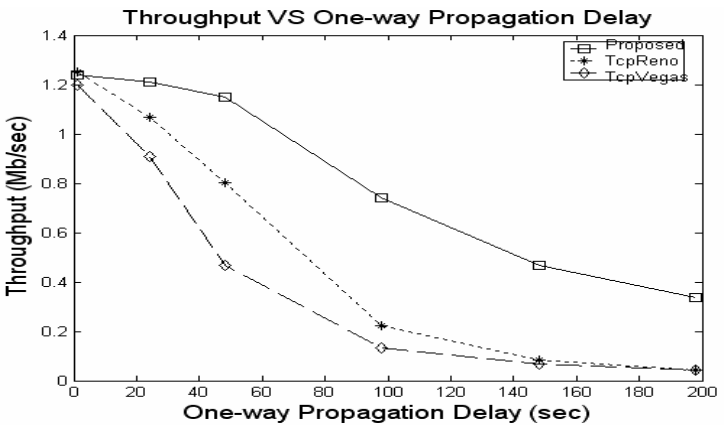

Fig2. Throughput vs. one-way propagation delay

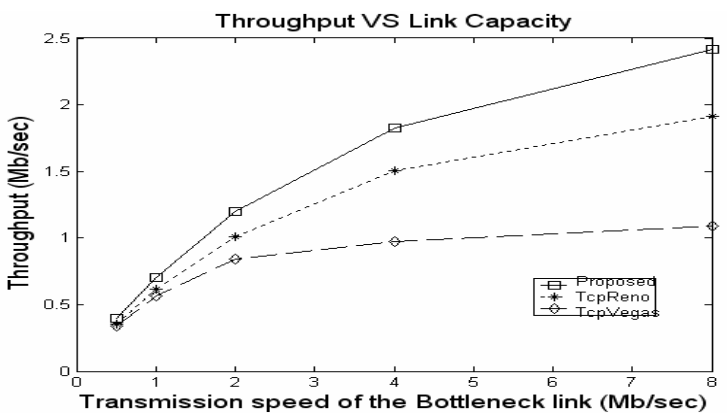

Fig3. Throughput vs. bottleneck capacity

\section{REFERENCES}

[1] R. Katz, “Adaptation and mobility in wireless information systems," IEEE Personal Communications, vol. 1, no. 1, 1994, pp. 6-17.

[2] C. Casetti, M. Gerla, S. Mascolo, M. Y. Sanadidi, R. Wang. “TCP Westwood: Bandwidth Estimation for Enhanced Transport over Wireless Links”, in Proceedings of Mobicom , Rome, Italy. July 2001. pp 287-297

[3]K. Lai and M. Baker, "Measuring Link Bandwidth Using a Deterministic Model of Packet Delay”, in Proc. ACM SIGCOM, Sept 2000, pp 283-294.

[4]M. Jain, C. Dovrolis, "End-to-End Available Bandwidth Methodology Dynamics and Relation with TCP Throughput.” Proc.ACM SIGCOMM, August 2002, pp. 295-308.

[5] B. Melander, M. Bjorkman, and P. Gunningberg, “A new end-to-end probing and analysis method for estimating bandwidth bottlenecks”, Global Internet Symposium, Dec 2000 [6] William C. Y. Lee. Mobile Communications Engineering: theory and Applications. McGraw-Hill Education, 1998

[7]Ns-2 network simulator (ver.2.) LBL, $U R L$ : http://www.mash.cs.berkley.edu/ns. 